\title{
Electric shock therapy for lung cancer: Taking palliation to the next level
}

\author{
Usman Ahmad, MD, ${ }^{a}$ and Michael Machuzak, MD $^{\mathrm{b}}$
}

\footnotetext{
From the a Section of Thoracic Surgery, Department of Cardiothoracic Surgery, Heart and Vascular Institute, and the ${ }^{b}$ Section of Interventional Pulmonology, Department of Pulmonary Medicine, Respiratory Institute, Cleveland Clinic, Cleveland, Ohio.

Disclosures: Authors have nothing to disclose about commercial support.

Received for publication Jan 4, 2018; accepted for publication Jan 12, 2018; available ahead of print Feb 15, 2018.

Address for reprints: Usman Ahmad, MD, Department of Cardiothoracic Surgery, Heart and Vascular Institute, Cleveland Clinic, Cleveland, OH 44195 (E-mail: ahmadu@ ccf.org).

J Thorac Cardiovasc Surg 2018;155:2160-1

$0022-5223 / \$ 36.00$

Copyright (c) 2018 by The American Association for Thoracic Surgery

https://doi.org/10.1016/j.jtcvs.2018.01.023
}

Electric shock application to the brain induces transient seizure activity. This effect is used to treat major depression, catatonia, and schizophrenia, among other maladies. Although the exact mechanism of effect remains elusive; the therapeutic utility of the transient effects has been explored in other disorders, including malignancies. In fact, "electrochemotherapy" is increasingly being used. ${ }^{1}$

Transient increase in cell membrane permeability by application of electric pulse (reversible electroporation) has been shown to increase the delivery of therapeutic agents, whereas irreversible electroporation induces apoptosis. ${ }^{2}$ Electroporation is being studied in the treatment of head and neck tumors, melanoma and its cutaneous metastases, ${ }^{3}$ pancreatic tumors, ${ }^{4}$ and hepatobiliary ${ }^{5}$ tumors. In this issue of the Journal, Kodama and colleagues ${ }^{6}$ have described the feasibility of electroporation in the treatment of human bronchial epithelial and lung cancer cell lines.

Kodama and colleagues ${ }^{6}$ are to be lauded for identifying the right application for this technology in lung cancer space. Central unresectable obstructing tumors rarely get adequate palliation. Endobronchial therapeutic strategies, including laser treatment, débridement, cautery, and cryoablation, address only the visible tumor. Airway stenting provides only temporary relief and is not free of complications.

Local control of the extraluminal component relies on the efficacy of systemic treatment and external radiation. A locally delivered modality that can increase cellular uptake of the systemic drug in theory can provide better local control and palliation. This appears to provide a perfect opportunity for electroporation to prove an effective treatment. Tumor in the immediate vicinity of the endobronchial catheter is more likely to undergo irreversible electroporation and subsequent apoptosis. Surrounding, more distant tumor areas undergo reversible electroporation, which may allow higher intracellular concentration of cytotoxic agents, leading to a similar end result. This is an exciting direction of innovation in managing this particular problem.

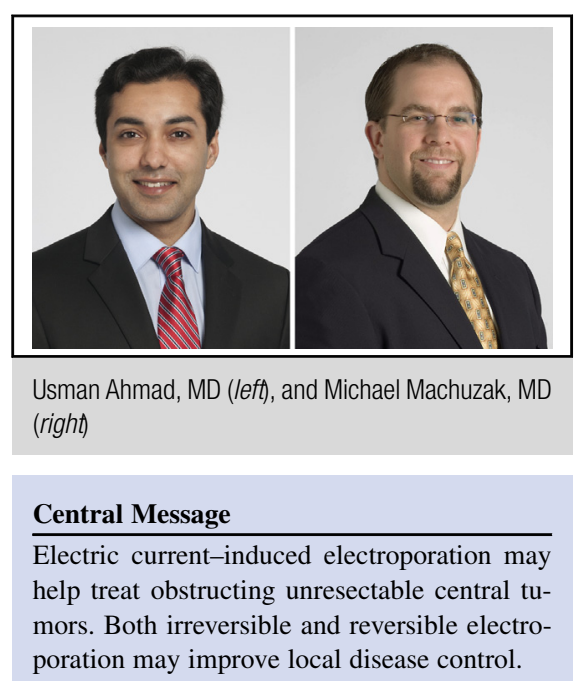

See Article page 2150.

The evidence is far from being conclusive, however, that the technology can be used in treating patients with lung cancer. Kodama and colleagues ${ }^{6}$ did show in their in vitro model that electroporation occurred in a bronchial epithelial cell line as well as in tumor cell lines. This electroporation increased the cytotoxic effects of cisplatin and gefitinib. The bronchial cells were more resilient to apoptosis from irreversible electroporation than were the tumor cells. It is important to note, however, that the in vivo reproducibility of both these findings in human patients remains to be demonstrated. In addition, the confounding effect of current-induced hyperthermia on increasing permeability is also unclear. Interestingly, much as in radiation planning, the area and size of effect of electroporation can be well simulated.

The effect of electroporation and the ability to simulate that effect accurately were both confirmed in a porcine model. It would be interesting to study the effect of electroporation in an actual animal model of lung cancer. Kodama and colleagues ${ }^{6}$ might consider using a xenograft tumor mouse model with percutaneous insertion of the electric probe into the tumor.

In their porcine model, Kodama and colleagues ${ }^{6}$ have elegantly used computed tomography to show the effect of electric current on bronchial and vascular changes. Posttreatment scans showed reductions in diameter of both bronchi and veins; however, this reversed at 2 to 4 hours after treatment. No treatment-related changes were noted on 
imaging at days 7 and 28. Pathologic evaluation similarly showed no granulation or remodeling at day 28 .

These findings are encouraging and certainly pave the way for more detailed study. As pointed out in the article, cardiac arrhythmias can be a serious complication, and gated application may therefore be the only way to deliver the electric pulse safely. Improvements in delivery catheters will be needed for bronchoscopic delivery. Percutaneous or thoracoscopic application should also be entertained.

In the last decade, there have been major advances in screening, local treatments, and systemic therapy for lung cancer. Our successes seem short lived, however, as we frequently find ourselves dealing with recurrent disease. Tumor biology continues to outsmart us, and it almost seems that lung cancer has a mind of its own. When we do not understand it and do not know what to do with it, why not do what the neurologists did, and give it electric shock treatment? Maybe we will find success, just as our colleagues in neurology have done.

\section{References}

1. Jiang C, Davalos RV, Bischof JC. A review of basic to clinical studies of irreversible electroporation therapy. IEEE Trans Biomed Eng. 2015;62:4-20.

2. Wagstaff PG, Buijs M, van den Bos W, de Bruin DM, Zondervan PJ, de la Rosette JJ, et al. Irreversible electroporation: state of the art. Onco Targets Ther 2016;9:2437-46.

3. Cerio R, Moir G. Palliative electrochemotherapy treatment of metastatic malignant melanoma. Br J Dermatol. 2017;176:1427.

4. Bhutiani N, Agle S, Li Y, Li S, Martin RC II. Irreversible electroporation enhances delivery of gemcitabine to pancreatic adenocarcinoma. J Surg Oncol. 2016;114: 181-6.

5. Langan RC, Goldman DA, D'Angelica MI, DeMatteo RP, Allen PJ, Balachandran VP, et al. Recurrence patterns following irreversible electroporation for hepatic malignancies. J Surg Oncol. 2017;115:704-10.

6. Kodama H, Vroomen LG, Uehsima E, Reilly J, Brandt W, Paluch LR, et al. Catheter-based endobronchial electroporation is feasible for the focal treatment of peribronchial tumors. J Thorac Cardiovasc Surg. 2018;155: 2150-9.e3. 